\title{
Some remarks on the Báez-Duarte criterion for the Riemann Hypothesis
}

\author{
Marek Wolf \\ Cardinal Stefan Wyszynski University \\ Faculty of Mathematics and Natural Sciences. College of Sciences \\ ul. Wóycickiego 1/3, Auditorium Maximum, (room 113) \\ PL-01-938 Warsaw, Poland \\ e-mail:m.wolf@uksw.edu.pl
}

Received: 4 March 2014; revised: 9 April 2014; accepted: 11 April 2014; published online: 25 April 2014

\begin{abstract}
In this paper we are going to describe the results of the computer experiment, which in principle can rule out validity of the Riemann Hypothesis (RH). We use the sequence $c_{k}$ appearing in the Báez-Duarte criterion for the RH and compare two formulas for these numbers. We describe the mechanism of possible violation of the Riemann Hypothesis. Next we calculate $c_{100000}$ with a thousand digits of accuracy using two different formulas for $c_{k}$ with the aim to disprove the Riemann Hypothesis in the case these two numbers will differ. We found the discrepancy only on the $996^{\text {th }}$ decimal place (accuracy of $10^{-996}$ ). The computer experiment reported herein can be of interest for developers of Mathematica and PARI/GP.
\end{abstract}

Key words: Riemann Hypothesis, zeta function, Baez-Duarte criterion

\section{INTRODUCTION}

With the advent of the computer era the computing machines have been used to prove mathematical theorems. The most spectacular examples of such a use of computers were proofs of the four color theorem [1, 2] and of the Kepler conjecture about sphere packing in three-dimensional Euclidean space [3]. It seems to be not possible to use computers for the proof of the Riemann Hypothesis (RH), but its refutation by numerical calculations seems to be plausible.

The Riemann Hypothesis says that the series

$$
\zeta(s)=\sum_{n=1}^{\infty} \frac{1}{n^{s}}, \quad(s=\sigma+i t, \quad \Re(s)>1)
$$

analytically continued to the complex plane in addition to trivial zeros $\zeta(-2 n)=0$ has nontrivial zeros $\zeta\left(\rho_{l}\right)=0$ in the critical strip $0<\Re(s)<1$ only on the critical line: $\Re\left(\rho_{l}\right)=\frac{1}{2}$ i.e. $\rho_{l}=\frac{1}{2}+i \gamma_{l}$, see e.g. the modern guide to the $\mathrm{RH}$ [4]. In the same book there is a review of failed attempts to prove $\mathrm{RH}$ in Chapter 8. Presently the requirement that the nontrivial zeros are simple $\zeta^{\prime}\left(\rho_{l}\right) \neq 0$ is often added.

The first use of computers in connection with RH was by Allan Turing checking whether the nontrivial zeros of $\zeta(s)$ have indeed real part $\frac{1}{2}$ [5]. Turing suspected that the RH is not true and the first counterexample is lying relatively low. Let us quote the sentence from the first page of his paper: "The calculation were done in an optimistic hope that a zero would be found off the critical line", but up to $t=1540$ Turing found that all zeros are on the critical line. The present record belongs to Xavier Gourdon [6] who has checked that all $10^{13}$ first zeros of the Riemann $\zeta(s)$ lie on the critical line. Andrew Odlyzko checked that RH is true in different intervals around $10^{20}$ [7], $10^{21}$ [8], $10^{22}$ [9], but his aim was not verifying the $\mathrm{RH}$ but rather provide evidence for conjectures that relate nontrivial zeros of $\zeta(s)$ to eigenvalues of random matrices. In fact, Odlyzko expressed the view that off critical line zeros could be encountered at least at $t$ of the order $10^{10^{10000}}$, see [10]. Asked by Derbyshire "What do you think about this darn Hypothesis? Is it true, or not?" Odlyzko 
replied: "Either it's true, or else it isn't". Also other famous mathematicians John E. Littlewood and Paul Turán did not believe RH is true. Aleksandar Ivić gave a few arguments against the truth of the $\mathrm{RH}$, see [4, ] and on arxiv [11].

There were several attempts to use computers to disprove some conjectures related to $\mathrm{RH}$ in the past. Sometimes it was sufficient to find a counterexample to the given hypothesis, sometimes the disproof was not direct. For example, the Haselgrove [12] disproved the Pólya's Conjecture stating that the function

$$
L(x):=\sum_{n \leq x} \lambda(n)
$$

satisfies $L(x) \leq 0$ for $x \geq 2$, where $\lambda(n)$ is Liouville's function defined by

$$
\lambda(n)=(-1)^{r(n)}
$$

where $r(n)$ is the number of, not necessarily distinct, prime factors in $n=p_{1}^{r_{1}} \cdots p_{\alpha(n)}^{r_{n}}$, with multiple factors counted according to their multiplicities: $r=r_{1}+\ldots+r_{n}$. From the truth of the Pólya Conjectures the RH follows, but the converse statement is not true. The Haselgrove proof was indirect, and in 1960 Lehman [13] found on the computer explicit counter-example: $L(906180359)=1$.

The next example is provided by the Mertens conjecture. Let $M(x)$ denote the Mertens function defined by

$$
M(x)=\sum_{n \leq x} \mu(n),
$$

where $\mu(n)$ is the Möbius function

$$
\mu(n)= \begin{cases}1 & n=1 \\ 0 & p^{2} \mid n \\ (-1)^{r} & n=p_{1} p_{2} \ldots p_{r}, \quad p_{i} \text { distinct }\end{cases}
$$

From

$$
|M(x)|<x^{\frac{1}{2}}
$$

again the RH would follow. However, in 1985 A. Odlyzko and $\mathrm{H}$. te Riele [14] disproved the Mertens conjecture, again not directly, but later it was shown by J. Pintz [15] that the first counterexample appears below $\exp \left(3.21 \times 10^{64}\right)$. The upper bound has since been lowered to $\exp \left(1.59 \times 10^{40}\right)$ [16].

Especially interesting is the value of the de BruijnNewman constant $\Lambda$, see e.g. $\$ 2.32$ (pp. 203-205) in [17]. Unconditionally $\Lambda \leq 1 / 2$ and the Riemann Hypothesis is equivalent to the inequality $\Lambda \leq 0$. The fascinating run for the best lower bound on $\Lambda$ ended with the value $\Lambda>$ $-2.7 \times 10^{-9}$ obtained by Odlyzko [18]. Such a narrow gap for values of $\Lambda$ being compatible with $\mathrm{RH}$ allowed Odlyzko to make the remark: "the Riemann Hypothesis, if true, is just barely true". However, Don Zagier [19] interpreted it as "the $\mathrm{RH}$, if false, is only slightly false".

In 1997 Xian-Jin Li proved [20], that Riemann Hypothesis is true iff the sequence:

$$
\lambda_{n}=\left.\frac{1}{(n-1) !} \frac{d^{n}}{d s^{n}}\left(s^{n-1} \ln \xi(s)\right)\right|_{s=1}
$$

where

$$
\xi(s)=\frac{1}{2} s(s-1) \Gamma\left(\frac{s}{2}\right) \zeta(s)
$$

fulfills:

$$
\lambda_{n} \geq 0 \quad \text { for } n=1,2, \ldots
$$

The explicit expression has the form:

$$
\lambda_{n}=\sum_{\rho}\left(1-(1-1 / \rho)^{n}\right) .
$$

K. Maślanka [21-23] has performed extensive computer calculations of these constants confirming (5) with large surplus.

Let us mention also the elementary Lagarias criterion [24]: to disprove the RH it suffices to find one $n$ that has so many divisors, that:

$$
\sum_{d \mid n} d>H_{n}+\exp \left(H_{n}\right) \ln \left(H_{n}\right),
$$

where $H_{n}=\sum_{k=1}^{n} 1 / k$ is the $n$-th harmonic number. The Lagarias criterion is not well suited for computer verification and in [25] Keith Briggs has undertook instead the verification of the Robin [26] criterion for RH:

$$
\mathrm{RH} \Leftrightarrow \sum_{d \mid n} d<e^{\gamma} n \ln \ln (n) \quad \text { for } n>5040
$$

For appropriately chosen $n$ Briggs obtained for the difference between r.h.s. and 1.h.s. of the above inequality value as small as $e^{-13} \approx 2.2 \times 10^{-6}$, hence again $\mathrm{RH}$ is very close to being violated.

In this paper we are going to propose a method which in principle can provide a refutation of the $\mathrm{RH}$. The idea is to calculate a number with very high accuracy (one thousand digits) in two ways: one without any knowledge on the zeros of $\zeta(s)$ and second using the explicit formula involving all $\rho_{l}$. Despite some estimation presented in Sect. 3 indicating that the discrepancy could be found merely at much higher than a thousand decimal places we performed the calculations in an optimistic hope that we will find the discrepancy between these two numbers, paraphrasing the sentence of Turing. There is a lot of number theoretic functions often defined in an elementary way being expressed also by the "explicit" formulas in terms of zeros of the $\zeta(s)$ function. Let us mention here the Chebyshev function

$$
\psi(x)=\sum_{n \leq x} \Lambda(n) \quad(=\ln (\operatorname{lcm}(2,3, \cdots\lfloor x\rfloor)),
$$

where the von Mangoldt function $\Lambda(n)$ is defined as

$$
\Lambda(n)= \begin{cases}\ln p & \text { for } n=p^{m} \\ 0 & \text { in other cases }\end{cases}
$$

The explicit formula reads, see eg. [27] (the term $\ln \left(1-\frac{1}{x^{2}}\right)$ comes from trivial zeros):

$$
\psi(x)=x-\ln (2 \pi)-\frac{1}{2} \ln \left(1-\frac{1}{x^{2}}\right)-\sum_{\text {nontr. zeros } \rho_{l}} \frac{x^{\rho_{l}}}{\rho_{l}}
$$


Also the Mertens's function has the explicit representation (the last term is comprising contribution from all trivial zeros) [27]:

$$
\begin{gathered}
\sum_{n \leq x} \mu(n)=\sum_{\substack{\text { nontr. } \\
\text { zeros } \rho_{l}}} \frac{x^{\rho_{l}}}{\rho_{l} \zeta^{\prime}\left(\rho_{l}\right)}-2 \\
-\sum_{n=1}^{\infty}(-1)^{n}\left(\frac{2 \pi}{x}\right)^{2 n} \frac{1}{(2 n) ! n \zeta(2 n+1)}
\end{gathered}
$$

The problem with these series is that they are extremely slow converging because the partial sums oscillate with amplitudes diminishing at very slow rates. For example $\psi(1000001)=999586.597 \ldots$, while from (9) summing over $5,549,728$ zeros gives $999587.15 \ldots$. thus relative error is 0.000055 .

In the computer experiment reported here we were able to get discrepancy less than $10^{-996}$ between the quantity calculated from the generic formula and from an explicit one summed over only 2600 nontrivial zeros computed with 1000 significant digits. This paper can be regarded as a continuation of our investigation reported in [28].

\section{THE BÁEZ-DUARTE CRITERION FOR THE RIEMANN HYPOTHESIS}

We begin by recalling the following representation of the $\zeta(s)$ function valid on the whole complex plane without $s=1$ found by Krzysztof Maślanka [29, 30]:

$$
\zeta(s)=\frac{1}{s-1} \sum_{k=0}^{\infty} \frac{\Gamma\left(k+1-\frac{s}{2}\right)}{\Gamma\left(1-\frac{s}{2}\right)} \frac{A_{k}}{k !}
$$

where $\Gamma(z)$ is the Euler gamma function and

$$
\begin{aligned}
A_{k}:=\sum_{j=0}^{k}(-1)^{j}\left(\begin{array}{l}
k \\
j
\end{array}\right)(2 j+1) \zeta(2 j+2) \equiv \\
\equiv \sum_{j=0}^{k}\left(\begin{array}{l}
k \\
j
\end{array}\right) \frac{(-1)^{j+1} 2^{2 j+1} B_{2 j+2} \pi^{2 j+2}}{(4 j+4) !} .
\end{aligned}
$$

Above we have used the fact that $\zeta(2 n)=$ $(-1)^{n-1} 2^{2 n-1} B_{2 n} \pi^{2 n} /(2 n)$ ! where $B_{2 n}$ are Bernoulli numbers.

The expansion (11) provides an example of the analytical continuation of (1) to the whole complex plane except $s=1$. Since $A_{k}$ tend to zero sufficiently fast as $k \rightarrow \infty$ the expansion (11) converges uniformly on the whole complex plane [31]. Based on the representation (11) Luis BáezDuarte in [32] proved that RH is equivalent to the statement that

$$
c_{k}=\mathcal{O}\left(k^{-3 / 4+\varepsilon}\right), \quad \forall \varepsilon>0
$$

where

$$
\begin{gathered}
c_{k}=\sum_{j=0}^{k}(-1)^{j}\left(\begin{array}{l}
k \\
j
\end{array}\right) \frac{1}{\zeta(2 j+2)} \equiv \\
\equiv \sum_{j=0}^{k}(-1)^{j+1}\left(\begin{array}{l}
k \\
j
\end{array}\right) \frac{(4 j+4) !}{2^{2 j+1} B_{2 j+2} \pi^{2 j+2}} .
\end{gathered}
$$

If additionally

$$
c_{k}=\mathcal{O}\left(k^{-3 / 4}\right)
$$

then all zeros of $\zeta(s)$ are simple. Báez-Duarte showed unconditionally (regardless of validity of the $\mathrm{RH}$ ) slower decrease $c_{k}=\mathcal{O}\left(k^{-1 / 2}\right)$. The plot of $c_{k}$ for $k=$ $1,2, \ldots, 1000000$ is presented in Fig. 1. We anticipate here that the formula (14) contains contribution from all zeros of $\zeta(s)$.

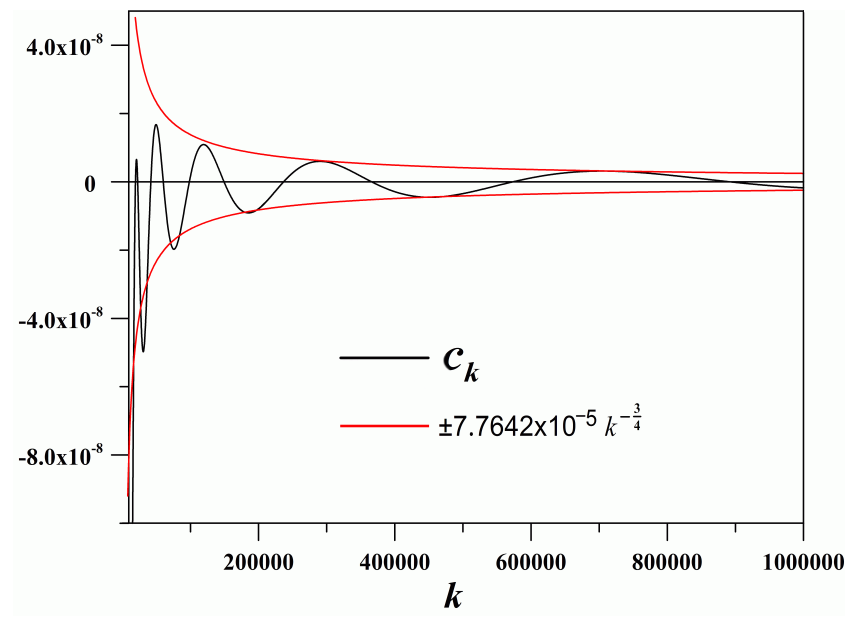

Fig. 1. The plot of the Báez-Duarte sequence $c_{k}$ for $k \in\left(1,10^{6}\right)$. The equation for the envelope was obtained from the explicit equation (27): for large $k$ the oscillating part $\tilde{c_{k}}$ is dominant and for $k>100000 c_{k}$ fits well between the red lines, for details see [28]

In [32] Báez-Duarte was able to express $c_{k}$ as a sum over zeros of $\zeta(s)$. The explicit formula for the sequence $c_{k}$ can be written as a sum of two parts: quickly decreasing with $k$ trend $\bar{c}_{k}$ arising from trivial zeros of $\zeta(s)$ and oscillations $\tilde{c}_{k}$ involving complex nontrivial zeros:

$$
c_{k}=\bar{c}_{k}+\tilde{c}_{k}
$$

Because the derivatives $\zeta^{\prime}(-2 n)$ at trivial zeros are known analytically:

$$
\zeta^{\prime}(-2 n)=\frac{(-1)^{n} \zeta(2 n+1)(2 n) !}{2^{2 n+1} \pi^{2 n}}
$$


Maślanka in [33] was able to give the closed expression for trend stemming from zeros $\rho_{n}=-2 n$ :

$$
\begin{aligned}
\bar{c}_{k} & =-\frac{1}{(2 \pi)^{2}} \\
& \times \sum_{m=2}^{\infty} \frac{\Gamma(k+1) \Gamma(m)}{\Gamma(k+m+1) \Gamma(2 m-1)} \frac{(-1)^{m}(2 \pi)^{2 m}}{\zeta(2 m-1)} .
\end{aligned}
$$

Báez-Duarte is skipping the trend $\overline{c_{k}}$ remarking only that it is of the order $o(1)$ (Remark 1.6 in [32]). It is an easy calculation to show (see [28]) that for large $k$

$$
\bar{c}_{k}=-\frac{(2 \pi)^{2}}{2 \zeta(3)} \frac{1}{k^{2}}+\mathcal{O}\left(k^{-3}\right),
$$

thus the dependence $c_{k}=\mathcal{O}\left(k^{-3 / 4}\right)$ in (13) is linked to the oscillating part $\tilde{c}_{k}$.

For $\tilde{c}_{k}$ Báez-Duarte gives the formula [32]:

$$
\tilde{c}_{k-1}=\frac{1}{2 k} \sum_{\rho_{l}} \operatorname{Res}\left(\frac{1}{\zeta^{\prime}(s) P_{k}(s / 2)} ; s=\rho_{l}\right),
$$

where

$$
P_{k}(s):=\prod_{r=1}^{k}\left(1-\frac{s}{r}\right)
$$

is the Pochhammer symbol. Assuming zeros of $\zeta(s)$ are simple we can write:

$$
\tilde{c}_{k-1}=\frac{1}{2 k} \sum_{\rho_{l}} \frac{1}{\zeta^{\prime}\left(\rho_{l}\right) P_{k}\left(\rho_{l} / 2\right)} .
$$

An appropriate order of summation over nontrivial zeros is assumed in (20) and (22), see [32, Theorem 1.5]. Because

$$
P_{k}(s)=\frac{(-1)^{k} \Gamma(s)}{\Gamma(k+1) \Gamma(s-k)}
$$

collecting in pairs $\rho_{l}$ and $\overline{\rho_{l}}$ we can convert (22) to the form:

$$
\tilde{c}_{k}=(-1)^{k+1} \Re\left(\sum_{\rho_{l}, \Im\left(\rho_{l}\right)>0} \frac{\Gamma(k+1) \Gamma\left(\frac{\rho_{l}}{2}-k-1\right)}{\Gamma\left(\frac{\rho_{l}}{2}\right) \zeta^{\prime}\left(\rho_{l}\right)}\right) .
$$

We have found that the numerical calculation of $P_{k}\left(\rho_{l} / 2\right)$ in PARI/GP directly from the above product (21) is much slower than the use of the $\Gamma(z)$ functions (23).

Báez-Duarte proves in [32, Lemma 2.2] that

$$
\lim _{k \rightarrow \infty} P_{k}(s) k^{s}=\frac{1}{\Gamma(1-s)}
$$

thus for large $k$ we can replace $k+1$ by $k$ and transform (22) in the following way:

$$
\begin{array}{r}
\tilde{c}_{k}=\frac{1}{2 k} \sum_{\rho_{l}} \frac{k^{\rho_{l} / 2}}{\zeta^{\prime}\left(\rho_{l}\right) P_{k}\left(\rho_{l} / 2\right) k^{\rho_{l} / 2}}= \\
=\Re\left(\sum_{\rho_{l}, \Im\left(\rho_{l}\right)>0} \frac{k^{\rho_{l} / 2-1} \Gamma\left(1-\frac{\rho_{l}}{2}\right)}{\zeta^{\prime}\left(\rho_{l}\right)}\right) .
\end{array}
$$

Now we assume the RH: $\rho_{l}=\frac{1}{2}+i \gamma_{l}$. Then we get for $\tilde{c}_{k}$ the overall factor $k^{-3 / 4}$ - the dependence following from $\mathrm{RH}$, see (13) - multiplied by oscillating terms:

$$
\tilde{c}_{k}=\frac{1}{k^{3 / 4}} \Re\left(\sum_{\gamma_{l}>0} \frac{k^{i \gamma_{l} / 2} \Gamma\left(\frac{3}{4}-\frac{i}{2} \gamma_{l}\right)}{\zeta^{\prime}\left(\frac{1}{2}+i \gamma_{l}\right)}\right) .
$$

Using the formula (6.1.45) from [34]:

$$
\lim _{|y| \rightarrow \infty} \frac{1}{\sqrt{2 \pi}}|\Gamma(x+i y)| e^{\frac{1}{2} \pi|y|}|y|^{\frac{1}{2}-x}=1
$$

assuming RH we obtain for large $\gamma_{l}>0$ :

$$
\left|\Gamma\left(\frac{3}{4} \mp \frac{i}{2} \gamma_{l}\right)\right| \approx \sqrt{2 \pi} e^{-\pi \gamma_{l} / 4}\left(\frac{\gamma_{l}}{2}\right)^{\frac{1}{4}},
$$

hence we get exponential decrease of summands in the sum (27) over nontrivial zeros giving $\tilde{c}_{k}$ and (27) is very fast convergent. Because of that if $\mathrm{RH}$ is true the sum (27) will be dominated by first zero $\gamma_{1}=14.13472514 \ldots$, which leads to the approximate expression (for details see [28]):

$$
\tilde{c}_{k}=\frac{A}{k^{\frac{3}{4}}} \sin \left(\phi-\frac{1}{2} \gamma_{1} \ln (k)\right)
$$

$$
A=7.775062 \ldots \times 10^{-5}, \quad \phi=2.592433 \ldots .
$$

For large $k$ the above formula (30) gives a very fast method for calculating quite accurate values of $c_{k}$, orders faster than (14).

In the following we will denote by $c_{k}^{g}$ the values of the Báez-Duarte sequence obtained from the generic formula (14)

$$
c_{k}^{g}=\sum_{j=0}^{k}(-1)^{j}\left(\begin{array}{l}
k \\
j
\end{array}\right) \frac{1}{\zeta(2 j+2)}
$$

and by $c_{k}^{e}$ the values obtained from explicit formula (16), i.e. in fact from (18) and (27) as no one zero off critical line is known:

$$
\begin{aligned}
& c_{k}^{e}=-\frac{1}{(2 \pi)^{2}} \sum_{m=2}^{\infty} \frac{\Gamma(k+1) \Gamma(m)}{\Gamma(k+m+1) \Gamma(2 m-1)} \\
& \times \frac{(-1)^{m}(2 \pi)^{2 m}}{\zeta(2 m-1)}+\frac{1}{k^{3 / 4}} \Re\left(\sum_{\gamma_{l}>0} \frac{k^{i \gamma_{l} / 2} \Gamma\left(\frac{3}{4}-\frac{i}{2} \gamma_{l}\right)}{\zeta^{\prime}\left(\frac{1}{2}+i \gamma_{l}\right)}\right) .
\end{aligned}
$$

\section{THE SCENARIO OF VIOLATION OF THE RIEMANN HYPOTHESIS}

The condition (15) means that the combination $k^{\frac{3}{4}} c_{k}$ should be contained between two paralel lines $\pm C$ for all $k$, where $C$ is the constant hidden in big- $\mathcal{O}$ in (15). The violation of the RH would manifest as an increase of the amplitude of the combination $k^{\frac{3}{4}} c_{k}$ and for sufficiently large $k$ (depending on $C$ ) the product $k^{\frac{3}{4}} c_{k}$ will escape outside the strip $\pm C$ (if RH is true we can take $C=A=7.7751 \ldots \times 10^{-5}$ ). We will discuss below the possible mechanism of violation of the inequality $c_{k}<C / k^{3 / 4}$ for the case of simple zeros of zeta function: $\zeta^{\prime}(s) \neq 0$. 
The derivatives $\zeta^{\prime}\left(\rho_{l}\right)$ in the denominator of (26) does not pose any threat to RH. First of all it does not depend on $k$, thus hypothetical extremely small values of $\zeta^{\prime}\left(\rho_{l}\right)$ will only change the constant hidden in big- $\mathcal{O}$ in (15). Second, this derivative is taking moderate values for zeros used by us: the smallest $\zeta^{\prime}\left(\frac{1}{2}+\gamma_{l}\right)$ was $0.032050162 \ldots$ at $\gamma_{1310}=$ $1771.212945 \ldots$ and the largest was $7.7852581838 \ldots$ at $\gamma_{1773}=2275.06866478 \ldots$. We remark that R.S. Lehman in [13] makes the conjecture:

$$
\frac{1}{\zeta^{\prime}\left(\rho_{l}\right)}=\mathcal{O}\left(\rho_{l}^{\nu}\right), \quad \text { where } \quad 0<\nu<1 .
$$

Some rigorous theorems about the possible large and small values of $\zeta^{\prime}\left(\rho_{l}\right)$ proved under the assumption of RH can be found in [35]. We checked that for first 5549728 nontrivial zeros of $\zeta^{\prime}\left(\rho_{l}\right)$ the largest derivative was 9.38127677 at $\gamma_{5376610}$ and the smallest was 0.001028760514 at $\gamma_{4161179}$.

Let us suppose there are some zeros of $\zeta(s)$ off critical line. Next let us assume that we can split the sum over zeros $\rho_{l}$ in (26) in two parts: one over zeros on the critical line and second over zeros off the critical line. This second sum should violate the overall term $k^{-3 / 4}$ present in the first sum. Let $\rho_{l}^{(o)}$ denote the zeros lying off the critical line ("o" stands for "off"): $\rho_{l}^{(o)}=\frac{1}{2} \pm \delta_{l}+i \gamma_{l}^{(o)}, \quad 0<\delta_{l}<1 / 2$ (as it is well known the nontrivial zeros are symmetric with respect to the critical line zero hence the combination $\pm \delta_{l}$ plus there are appropriate complex conjugate zeros below real axis). In the factor $\left|\Gamma\left(1-\frac{\rho_{l}}{2}\right)\right|$ the off-critical line zeros will lead only to the change of $\gamma_{l}^{\frac{1}{4}} \rightarrow \gamma_{l}^{\frac{1}{4} \mp \delta_{l} / 2}$ in (29) and in view of exponential decrease present in (29) the possible violation of RH in (26) will manifest through the terms $k^{\rho_{l} / 2-1}=k^{-\frac{3}{4} \pm \delta_{l} / 2+i \gamma_{l}^{(o)} / 2}$. The combination $1 / k^{\frac{3}{4}+\delta_{l} / 2}$ poses no problem as it leads to faster than required in (15) decrease of some terms in the sum for $\tilde{c_{k}}$. But the combination $1 / k^{\frac{3}{4}-\delta_{l} / 2}$ leads to violation of (15) and we want to elucidate how it can be detected. Say we want to compare $c_{k}^{g}$ with $c_{k}^{e}$ with accuracy $\epsilon$, where we are interested in values of $\epsilon$ of the order $\epsilon=10^{-10 \ldots 0}$. The expression for $c_{k}^{g}$ is a finite sum and we can in principle calculate its value in PARI with practically arbitrary exactness (however, for really large $k$ it can take years of CPU time). Although $c_{k}^{g}$ contains information coming from all zeros, to see influence of the first off the critical line zero the value of sufficiently large $k$ has to be examined. The sum for $c_{k}^{e}$ is infinite and we expect that to get accuracy $\epsilon$ we have to sum in (27) up to $l=L$ given by (as we skip $\left|\gamma_{l}\right|^{1 / 4}$ we will skip also $\pi / 4$ as our consideration are not rigorous in general):

$$
\epsilon \approx e^{-\gamma_{L}}, \text { hence } \gamma_{L} \approx-\ln (\epsilon) .
$$

Because values of the imaginary parts $\gamma_{l}^{(o)}$ of the hypothetical zeros off critical line should be extremely large, perhaps even as large as $10^{10^{10000}}$, see [10], we suppose that $\gamma_{L}<\gamma_{l}^{(o)}$. The contribution of $\gamma_{l}^{(o)}$ is present in $c_{k}^{g}$, but will not be present in the explicit sum for $c_{k}^{e}$ cut at $L$. To detect discrepancy between $c_{k}^{g}$ and $c_{k}^{e}$ larger than assumed accuracy $\epsilon$ sufficiently large value of $k=K$ is needed. The point is that $k^{3 / 4} c_{k}^{g}$ will escape outside the strip $\pm C$ for sufficiently large $k=K$ and the value of such $K$ we can estimate analyzing the explicit formula for $\tilde{c_{k}}$.

We can estimate value of the index $K$ from the requirement that the term $K^{\delta_{l} / 2}\left(K^{-3 / 4}\right.$ is present in front of the sum for $\tilde{c_{k}}$ ) will defeat the smallness of the term $\Gamma\left(3 / 4-\gamma_{l}^{(o)} / 2\right)$ and together their product will overcome the first summand in (26) corresponding to $\gamma_{1}$. In other words in the series (26) all terms up to $\gamma_{l}^{(o)}$ monotonically and fast decrease but the terms corresponding to zeros off the critical line can be made arbitrary large for sufficiently large $k$. Instead of (29) now will have:

$$
\begin{gathered}
\left|\Gamma\left(\frac{3}{4} \mp \frac{\delta_{l}}{2} c-i \gamma_{l}^{(o)}\right)\right| \approx \\
\approx \sqrt{2 \pi} e^{-\pi \gamma_{l}^{(o)} / 4}\left(\frac{\gamma_{l}^{(o)}}{2}\right)^{-1 / 4 \pm \delta_{l} / 2} .
\end{gathered}
$$

The condition for such a $K$ is roughly:

$$
K^{\delta_{l} / 2} e^{-\gamma_{l}^{(o)}}>C \text { thus } K>C^{\prime} e^{2 \gamma_{l}^{(o)} / \delta_{l}} .
$$

Because $\delta_{l}$ can be arbitrarily close to zero and, as we expect, $\gamma_{l}^{(o)}$ is very large the value of $K$ will be extremely huge - larger than the famous Skewes number and will look something like $10^{10}$. Thus it is practically impossible to disprove RH by comparing $c_{k}^{g}$ and $c_{k}^{e}$. Maślanka presented in [33] a discussion of possible violation of (15) and he also came to the pessimistic conclusion that disproving RH by comparing $c_{k}^{g}$ and $c_{k}^{e}$ is "far beyond any numerical capabilities", see pp. 7-8. We wanted to find agreement between $c_{100000}^{g}$ and $c_{100000}^{e}$ within one thousand digits and to our surprise the first attempt to calculate $c_{100000}^{e}$ resulted in the difference already on the $87^{\text {th }}$ place. We started to struggle with numerical problems to improve the accuracy and finally we got 996 digits of $c_{100000}^{g}$ and $c_{100000}^{e}$ the same.

\section{THE COMPUTER EXPERIMENT}

The idea of the experiment is to calculate to high precision the values of $c_{100000}^{g}$ and $c_{100000}^{e}$ and try to find a discrepancy between them. We calculated one value $c_{100000}^{g}$ from the generic formula (14) which contains contribution from all zeros of $\zeta(s)$, even hypothetical zeros with $\Re\left(\rho_{l}\right) \neq \frac{1}{2}$. Because $\zeta(2 n)$ very quickly tend 1 to get the firm value of $c_{k}$ it is necessary to perform calculations with many digits accuracy. An additional problem is fast growing of binomial symbols. We performed a calculation of $c_{100000}^{g}$ using the free package PARI/GP [36]. This package allows to perform very fast computations practically of arbitrary precision. We set precision to 100000 decimals and below in Table I are the partial sums of (14) recorded after summation 
of $10000,20000, \ldots 100001$ terms. In the middle of computations the partial sums for $c_{100000}^{g}$ were of the order $10^{30100}$ to drop finally to $1.609757993 \ldots \times 10^{-9}$ after summing up all 100001 terms. Separately we repeated these calculations with precision set to 150000 places and we found the difference only from the $69900^{\text {th }}$ place. Because the sum (14) is finite we got accurate say 50000 digits from this generic formula for $c_{100000}^{g}$. This was an easy part which took approximately 14 hours for precision 100000 decimals and over 20 hours for precision 150000 digits on the AMD Opteron 2.6 $\mathrm{GHz} 64$ bits processor.

Now we turn to the calculation of $c_{100000}^{e}$ from the explicit formulas (18) and (27), which are infinite sums. It is important not to make the replacement $k+1 \rightarrow k$ even for $k=100000$ if we want to get accuracy of the order of 1000 digits. The series in (18) decreases very fast to zero and it is very easy to get arbitrary number of digits of $\bar{c}_{k}$ using the PARI/GP procedure sumalt.

Next we want to calculate $\tilde{c}_{k}$ from (27) with 1000 digits accuracy. From $e^{-\pi \gamma_{l} / 4}=10^{-1000}$ we get $\gamma_{l} \approx 2931.7$ and a glance at the list of zeros of $\zeta(s)$ (e.g. [37]) gives $l=2402$ because $\gamma_{2402}=2931.0691 \ldots$ First 100 zeros of $\zeta(s)$ accurate to over 1000 decimal places we took from [37]. Next 2500 zeros of $\zeta(s)$ and derivatives $\zeta^{\prime}\left(\rho_{l}\right)$ with precision 1000 digits we decided to calculate using the built in Mathematica v.7 procedures ZetaZero [m] and numerical differentiation ND $[\ldots]$. After a few days we got the values of $\gamma_{l}$ and $\zeta^{\prime}\left(\rho_{l}\right)$. We checked using PARI/GP that these zeros were accurate within at least 996 places in the sense that in the worst case $\left|\zeta\left(\rho_{l}\right)\right|<10^{-996}, l=1,2, \ldots, 2600$. The formulas (27) and (18) were implemented in PARI with precision set to 1000 digits and to our surprise we found that the values of $c_{100000}^{g}$ and $c_{100000}^{e}$ coincide only up to $87^{\text {th }}$ place:

$$
10^{-86}>\left|\frac{c_{100000}^{g}}{c_{100000}^{e}}-1\right|>10^{-87} .
$$

Tab. 1. The partial sums of (14) recorded after summation of 10000, 20000,... 100001 terms

\begin{tabular}{c|c}
\hline$n$ & $\sum_{j=0}^{n}(-1)^{j}\left(\begin{array}{c}100000 \\
j\end{array}\right) \frac{1}{\zeta(2 j+2)}$ \\
\hline 10000 & $5.65168726144550 \times 10^{14115}$ \\
\hline 20000 & $4.00927204946289 \times 10^{21729}$ \\
\hline 30000 & $6.08771775660005 \times 10^{26526}$ \\
\hline 40000 & $5.17938759373151 \times 10^{29225}$ \\
\hline 50000 & $1.26030418446100 \times 10^{30100}$ \\
\hline 60000 & $3.45292506248767 \times 10^{29225}$ \\
\hline 70000 & $2.60902189568574 \times 10^{26526}$ \\
\hline 80000 & $1.00231801236572 \times 10^{21729}$ \\
\hline 90000 & $6.27965251271723 \times 10^{14114}$ \\
\hline 100000 & $1.60975799392038 \times 10^{-9}$ \\
\hline
\end{tabular}

The arguments given earlier in Sect. 3 suggested that no discrepancy should be found between $c_{k}^{g}$ and $c_{k}^{e}$ for $k=$ 100000 hence we paid attention to the numerical inaccuracies in the computation of the derivative $\zeta^{\prime}\left(\rho_{l}\right)$ as a possible explanation. We stress here that $c_{k}^{g}$ contains information on all zeros of $\zeta(s)$, even off the critical line, while the sum for $c_{k}^{e}$ is truncated, thus ruling out numerical reasons or mistakes of the program should disprove the Riemann Hypothesis. We played with different options 20, 30, 40 terms and WorkingPrecision in ND [... ], but finally we got only the moderate improvement: the difference between $c_{100000}^{g}$ and $c_{100000}^{e}$ shifted to $105^{\text {th }}$ place:

$$
10^{-105}<\left|\frac{c_{100000}^{g}}{c_{100000}^{e}}-1\right|<10^{-104}
$$

Because in (27) gamma functions can be calculated in PARI with practically arbitrary digits of accuracy, the only way to improve accuracy of calculation of $c_{100000}^{e}$ is to find a reliable method for calculating $\zeta^{\prime}(s)$ with certainty that say all 1000 digits are correct. From (1) it is easy to obtain the modified expression for zeta:

$$
\zeta(s)=\frac{1}{1-2^{1-s}} \sum_{n=1}^{\infty} \frac{(-1)^{n-1}}{n^{s}} .
$$

By the Leibniz test for alternating series the above sum converges for $\Re(s)=\sigma>0$. Then it uniformly converges on every compact subset lying interior to the half-plane of convergence (see eg. [38, Th. 11.11 and Th. 11.12]) and thus can be differentiated term by term:

$$
\begin{aligned}
& \zeta^{\prime}(s)=-\frac{\ln (2) 2^{1-s}}{\left(1-2^{1-s}\right)^{2}} \sum_{n=1}^{\infty} \frac{(-1)^{n-1}}{n^{s}} \\
& +\frac{1}{1-2^{1-s}} \sum_{n=1}^{\infty} \frac{(-1)^{n} \ln (n)}{n^{s}} .
\end{aligned}
$$

PARI contains the numerical routine sumalt for summing infinite alternating series in which an extremely efficient algorithm of Cohen, Villegas and Zagier [39] is implemented. As these authors point out on p. 6, their algorithm works even for series like (36) with $s$ complex - (36) is alternating only when $s \in \mathbb{R}$. We used this routine sumalt outside scope of its applicability with success to calculate $\zeta^{\prime}\left(\rho_{l}\right)$ from (37) with precision set to 1000 digits and then calculated $c_{100000}^{e}$ from (27) and (18). The result was astonishing: the difference between $c_{100000}^{e}$ and $c_{100000}^{g}$ appeared on the $625^{\text {th }}$ place:

$$
10^{-625}<\left|\frac{c_{100000}^{g}}{c_{100000}^{e}}-1\right|<10^{-624}
$$

Because we expected that a possible violation of RH should manifest at much larger $k$ we were looking for a way to still improve the accuracy of $\zeta^{\prime}\left(\rho_{l}\right)$. We decided to make a frenzy think: we calculated again $\zeta^{\prime}\left(\rho_{l}\right)$ using sumalt with zeros having 1000 digits but this time with precision set to 2000 (however, values of $\zeta^{\prime}\left(\rho_{l}\right)$ were stored only with 1000 digits). Thus the aim was to enlarge the number of terms 
summed in (37), or in fact the number of iteration performed inside sumalt until the prescribed accuracy was attained. After 18 hours on AMD Opteron 2.6 GHz we got the results. And now bingo! The first 996 digits of $c_{100000}^{g}$ and $c_{100000}^{e}$ were the same:

$$
10^{-996}<\left|\frac{c_{100000}^{g}}{c_{100000}^{e}}-1\right|<10^{-995} .
$$

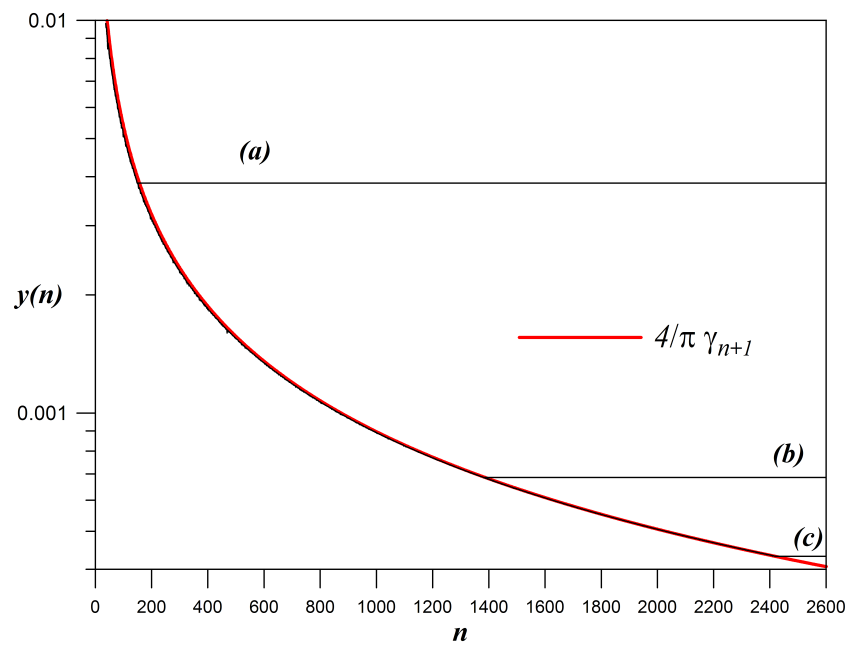

Fig. 2. The plot of $y(n)$ for $c_{100000}^{e}$ obtained by three methods. The plot $(a)$ is the best result obtained with Mathematica with the procedure ND $[\ldots]$ with the option terms $=30$ and WorkingPrecision=1000. The accuracy was 105 digits and the curve $(a)$ departs from the red line at the zero $\gamma_{149}=317.73480594237 \ldots$ for which $\exp \left(-\pi \gamma_{149} / 4\right)=$ $4.193107483 \times 10^{-109}$. The plot $(b)$ was obtained with PARI from the formula (37) using the procedure sumalt with precision set to 1000 digits. The $y(n)$ reaches plateau $0.0006856926750 \ldots$ at zero $\gamma_{1412}=1884.00577834967 \ldots$, for which $\exp \left(-\pi \gamma_{1412} / 4\right)=$ $2.391868726 \times 10^{-643}$. The curve $(c)$ is the same as $(b)$ but derivatives $\zeta^{\prime}\left(\frac{1}{2}+i \gamma_{l}\right)$ were calculated with sumalt and precision set to 2000 digits. Curve $(c)$ reaches the plateau $0.00043191361 \ldots$ at the zero $\gamma_{2430}=2960.033617812 \ldots$ for which $\exp \left(-\pi \gamma_{2430} / 4\right)=2.298783954 \times 10^{-1010}$
Because we got the precision (39) it is a posteriori proof that 1000 digits of derivatives $\zeta^{\prime}\left(\rho_{l}\right)$ were calculated correctly from (37) by the PARI procedure sumalt with precision set to 2000 digits.

In Fig. 2 we present a summary of these computer calculations. Since it is not possible to plot using standard plotting software as small values as $10^{-600}$ on the $y$-axis we present in Fig. 2 the following quantity measuring the distance from $c_{100000}^{g}$ to the partial sums over zeros $\gamma_{l}$ in (27) and decreasing with a number of zeros included in the sum:

$$
\begin{aligned}
& y(n)= \\
= & \left(\ln \left(\left|\frac{1}{k^{3 / 4}} \Re\left(\sum_{l=1}^{n} \frac{k^{i \gamma_{l} / 2} \Gamma\left(\frac{3}{4}-\frac{i}{2} \gamma_{l}\right)}{\zeta^{\prime}\left(\frac{1}{2}+i \gamma_{l}\right)}\right)-c_{k}^{g}\right|\right)\right)^{-1}
\end{aligned}
$$

where $k=100000$ and the absolute value is necessary as the differences between successive approximants to $c_{100000}^{e}$ and $c_{100000}^{g}$ change sign erratically. The consecutive terms in the series (27) behave like $\mathrm{e}^{-\pi \gamma_{l} / 4}$ hence we expect that $y(n)$, by analogy with well known property of alternating series with decreasing terms, should behave like the first discarded term:

$$
y(n) \sim \frac{4}{\pi \gamma_{n+1}} .
$$

Fig. 2 confirms these considerations: initially $y(n)$ for all curves follows the prediction (41) and starting with $n$ for which the values of the $\zeta^{\prime}\left(\rho_{n}\right)$ are incorrect adding further terms does not improve accuracy. Because of the exponential decrease of $\Gamma\left(\frac{3}{4}-\frac{\gamma_{l}}{2}\right)$ the contribution of further terms is suppressed and horizontal lines in Fig. 2 are determined by the first $\gamma_{n}$ corresponding to the bad value of the derivative $\zeta^{\prime}\left(\rho_{n}\right)$.

\section{FINAL REMARKS}

Although we have reached the agreement between $c_{100000}^{g}$ and $c_{100000}^{e}$ up to 996 places:

$$
\begin{aligned}
& c_{100000}^{g}=1.60975799 \ldots \longleftarrow 980 \text { digits } \longrightarrow \ldots 2913696 \underline{30140} \times 10^{-9} \\
& c_{100000}^{e}=1.60975799 \ldots \longleftarrow 980 \text { digits } \longrightarrow \ldots 2913696 \underline{29833} \times 10^{-9}
\end{aligned}
$$

it means nothing about the validity of the RH. Refutation of the RH by computer methods seems to be as difficult as the analytical proof of its validity. We need to find an example of the quantity which can be expressed in two ways: one without the zeta zeros and the second formula containing contributions from all zeta zeros so that there is strong sensitivity on the location of high zeros. Then perhaps it can be possible to overthrow RH using a computer. As a possible precaution let us mention the paper "Strange Series and High Precision Fraud" written by Borwein brothers [40]. In this paper we found a few striking examples of the approximate equalities correct to numerous digits which finally are not identities. The most fraudulent is the following

$$
\sum_{n=1}^{\infty} \frac{\left\lfloor n e^{\pi \sqrt{163 / 9}}\right\rfloor}{2^{n}} \doteq 1280632
$$

which is valid up to accuracy at least $10^{-450,000,000}$ but is not an identity. 
In the end let us remark that the most accurate experiment in physics is the measurement of the ratio of the electric charge of the electron $e^{-}$to the charge of the proton $e^{+}$ which is known to be something like $e^{-} / e^{+}=-1 \pm 10^{-20}$, see [41]. Physicists believe that $e^{-}=-e^{+}$exactly.

\section{Acknowledgments}

I would like to thank Prof. Keith Briggs, Prof. Aleksandar Ivić, Prof. Krzysztof Maślanka, Prof. Filip Saidak and Prof. Don Zagier for comments and remarks.

\section{References}

[1] K. Appel and W. Haken, Every planar map is four colorable. Part I. Discharging, Illinois J. Math., pages 429-490, (1977).

[2] K. Appel and W. Haken, Every planar map is four colorable. Part II. Reducibility, Illinois J. Math., pages 491-567, (1977).

[3] T.C. Hales, The Kepler Conjecture, the series of papers: math.MG/9811071, math.MG/9811072, math.MG/ 9811079, math.MG/9811079, for current status see: http://sites.google.com/site/thalespitt/, http://www.math.pitt. edu/ thales/kepler98/.

[4] P. Borwein, S. Choi, B. Rooney, and A. W eirathmueller, The Riemann Hypothesis: A Resource For The Afficionado And Virtuoso Alike, p. 137, Springer Verlag, 2007.

[5] A.M. Turing, Some calculations of the Riemann zetafunction, Proc. London Math. Soc. (3) 3, 99-117, (1953).

[6] X. Gourdon, The $10^{13}$ First Zeros of the Riemann Zeta Function, and Zeros Computation at Very Large Height, Oct. 24, 2004, http://numbers.computation.free.fr/Constants/Miscellaneous/zetazeros1e13-1e24.pdf.

[7] A.M. Odlyzko, The $10^{20}$-th zero of the Riemann zeta function and 175 million of its neighbors, 1992 revision of 1989 manuscript.

[8] A.M. Odlyzko, The $10^{21}$-st zero of the Riemann zeta function, Nov. 1998 note for the informal proceedings of the Sept. 1998 conference on the zeta function at the Edwin Schroedinger Institute in Vienna.

[9] A.M. Odlyzko, The $10^{22}$-nd zero of the Riemann zeta function, In M. van Frankenhuysen and M. L. Lapidus, editors, Dynamical, Spectral, and Arithmetic Zeta Functions, number 290 in Amer. Math. Soc., Contemporary Math. series, pages 139-144, 2001.

[10] J. Derbyshire, Prime Obsession. Bernhard Riemann and the greatest unsolved problem in mathematics, p. 358, Joseph Henry Press, Washington, 2003.

[11] A. Ivic̀, On some reasons for doubting the Riemann hypothesis, arXiv:math/0311162, (November 2003).

[12] C.B. Haselgrove, A Disproof of a Conjecture of Polya, Mathematika 5, 141-145, (1958).

[13] R.S. Lehman, On Liouville's Function, Math. Comput. 4, 311-320, (1960).

[14] A.M. Odlyzko and H.J.J. te Riele, Disproof of the Mertens Conjecture, J. Reine Angew. Math. 357, 138-160, (1985).

[15] J. Pintz, An effective disproof of the Mertens conjecture, Asterisque 147-148, 325-333, (1987).

[16] T. Kotnik and H. te Riele, The Mertens Conjecture Revisited, In 7-th ANTS, volume 4076 of Lecture Notes in Computer Science, pages 156-167, 2006.

[17] S.R. Finch, Mathematical Constants, Cambridge University Press, 2003.
[18] A.M. Odlyzko, An improved bound for the de BruijnNewman constant, Numerical Algorithms 25(1), 293-303, (2000).

[19] D. Zagier, e-mail from 14 October 2009.

[20] X.J. Li, The Positivity of a Sequence of Numbers and the Riemann Hypothesis, Journal of Number Theory 65(2), 325-333, (1997).

[21] K. Maślanka, An Explicit Formula Relating Stieltjes Constants and Li's Numbers, 2004, http://xxx.lanl.gov/abs/ 0406312

[22] K. Maślanka, Li’S Criterion For The Riemann Hypothesis - Numerical Approach, Opuscula Mathematica 24, 103-114, (2004).

[23] K. Maślanka, Effective method of computing Li's coefficients and their properties, 2004, http://xxx.lanl.gov/abs/0402168.

[24] J.C. Lagarias, An elementary problem equivalent to the Riemann Hypothesis, Amer. Math. Monthly 109, 534-543, (2002).

[25] K. Briggs, Abundant Numbers and the Riemann Hypothesis, Experimental Mathematics 15, Number 2, 251-256, (2006).

[26] G. Robin, Grandes valeurs de la fonction somme des diviseurs et Hypothèse de Riemann, J. Math. Pures Appl. (9)63(2), 187-213, (1984).

[27] E.C. Titchmarsh, The Theory of the Riemann Zeta-function, The Clarendon Press Oxford University Press, New York, second edition, 1986, Edited and with a preface by D. R. Heath-Brown.

[28] M. Wolf, Evidence in favor of the Baez-Duarte criterion for the Riemann Hypothesis, Computational Methods in Science and Technology 14, 47, (Nov 2008).

[29] K. Maślanka, A hypergeometric-like Representation of Zetafunction of Riemann, Cracow Observatory preprint no. 1997/60, 1997, posted at arXiv: math-ph/0105007, (2001), http://xxx.lanl.gov/abs/math/0105007.

[30] K. Maślanka, The Beauty of Nothingness: Essay on the Zeta Function of Riemann, Acta Cosmologica XXIII-1, 13-17, (1998).

[31] L. Baez-Duarte, On Maslanka's representation for the Riemann zeta-function, International Journal of Mathematics and Mathematical Sciences 2010, 1-9, (2010).

[32] L. Báez-Duarte, A sequential Riesz-like criterion for the Riemann Hypothesis, International Journal of Mathematics and Mathematical Sciences 2005(21), 3527-3537, (2005).

[33] K. Maślanka, Baez-Duarte's Criterion for the Riemann Hypothesis and Rice's Integrals, math.NT/0603713, (2006).

[34] M. Abramowitz and I.A. Stegun, Handbook of Mathematical Functions with Formulas, Graphs, and Mathematical Tables, Dover, New York, ninth Dover printing, tenth GPO printing edition, 1964.

[35] N. Ng, Extreme values of $\zeta^{\prime}(\rho)$, arXiv:math/0706.1765v1 [math.NT], (2007).

[36] The PARI Group, Bordeaux, PARI/GP, version 2.3.2, 2008, available from http://pari.math.u-bordeaux.fr/.

[37] A.M. Odlyzko, Tables of zeros of the Riemann zeta function, http://www.dtc.umn.edu/ odlyzko/zeta_tables/index.html.

[38] T.M. Apostol, Introduction to Analytic Number Theory, Springer-Verlag, New York, 1976, Undergraduate Texts in Mathematics.

[39] H. Cohen, F.R. Villegas, and D. Zagier, Convergence acceleration of alternating series, Experiment. Math. 9(1), 3-12, (2000).

[40] J.M. Borwein and P.B. Borwein, Strange Series and High Precision Fraud, The American Mathematical Monthly 99(7), 622-640, (1992).

[41] C.S. Unnikrishnan and G.T. Gillies, The electrical neutrality of atoms and of bulk matter, Metrologia 41(5), 125-135, (2004). 


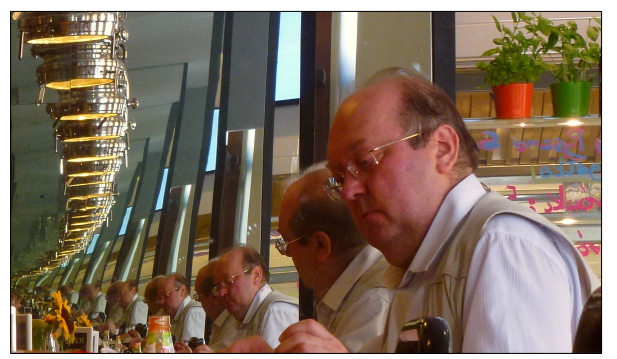

Marek Wolf, obtained $\mathrm{PhD}$ degree in physics (1982) and habilitation (1993) at the Wroclaw University, where he was employed till 2011. Since 1984 he has been doing computer experiments in physics and mathematics. In 1991 and 1993 he was a research fellow at the Center for Polymer Studies at the Boston University. Since 2011 he is a member of the Faculty of Mathematics and Natural Sciences, College of Sciences at the Cardinal Stefan Wyszynski University in Warsaw. His hobby is photography. 